\title{
AUTOCORRELATIVE ANALYSIS OF THE BRIGHTNESS OF IRREGULAR AND \\ SEMI-REGULAR VARIABLE STARS
}

\author{
F. I. LUKATSKA YA
}

Main Astronomical Observatory, Goloseevo, Kiev, U.S.S.R.

\begin{abstract}
This paper gives an introduction to the use of autocorrelative analyses in the case of irregular and semi-regular brightness variations.
\end{abstract}

The autocorrelative function of the brightness of a variable star is connected directly with the process of light variability. It is also a source of ideas about the mechanism causing it. Therefore it is natural that such functions are often calculated in the light analysis of irregular and semi-regular variable stars. Series of observations equispaced in time are generally used for such calculations. They are obtained during one night or during one observational season. The maximal autocorrelative delay must be less than an hour in the first case and less than 20-30 days in the second case because it must be several times smaller than the duration of the series.

One can calculate the autocorrelative functions for series with gaps. The series duration can reach some years and the maximal autocorrelative delay is only a few times smaller in this case. It is necessary only that the main statistical characteristics of the process be present in the time series with gaps.

Let us have a series of observed magnitudes of a variable celestial object assumed to be stationary and ergodic. This assumption must be based on the equality of mean magnitudes for two halves of the series. First we must choose a time unit for the analysis. It is equal to the minimal autocorrelative delay $(\tau)$. If the time unit is equal to the minimal difference of moments of observations, the series is analysed in a given form. If it is larger the magnitudes must be averaged for time intervals equal to the time unit. In all cases the time unit must be such that the series of averaged magnitudes will have a long duration and a large number of values.

Then we construct a series $u_{i}=m_{i}-\bar{m}$, where $m_{i}$ is the averaged magnitude and $\bar{m}$ is the mean magnitude. Let us write the autocorrelative function in a usual form

$$
r_{\tau}=\frac{\sum_{i=1}^{n_{\tau}} u_{i} u_{i+\tau}}{\sqrt{\sum_{i=1}^{n_{\tau}} u_{i}^{2} \sum_{i=1}^{n_{\tau}} u_{i+\tau}^{2}}}
$$

$n_{\tau}$ is equal to $N-\tau$ for series with $N$ values without gaps. Let us designate the absent terms in $u$ series as $u_{i}^{\prime}$ and $u_{i+\tau}^{\prime}$. Since the series is large, we can suppose that

$$
\overline{u_{i}^{2}}=\overline{u_{i}^{\prime 2}} \quad \overline{u_{i+\tau}^{2}}=\overline{u_{i+\tau}^{\prime 2}} \quad \overline{u_{i} u_{i+\tau}}=\overline{u_{i}^{\prime} u_{i+\tau}^{\prime}}
$$


Then one carries out the calculations using formula (1) only with such $u_{i}$ which have $u_{i+\tau}$ terms. Therefore $n_{\tau}$ is equal to the number of such $u_{i}$ for each $\tau$. For an irregular variable $n_{\tau}$ must be about 1000 and the duration of the series must be on the order of 1000 days. It follows that the characteristic time of irregular variability can be on the order of 100 days.

It is seen from (1) and (2) that $r_{\tau}$ so calculated is equal to $r_{\tau}$ for the full series of the same duration. Therefore the power spectral function can be calculated from the obtained $r_{\tau}$ in the usual manner.

$r_{\tau}$ were calculated in the described way for more than 50 irregular and semi-regular stars, mostly for visual series (Campbell, 1938, 1940, 1945, 1948; Lukatskaya, 1969, 1973; Kovalchuk et al., in press). The obtained autocorrelative functions are of three
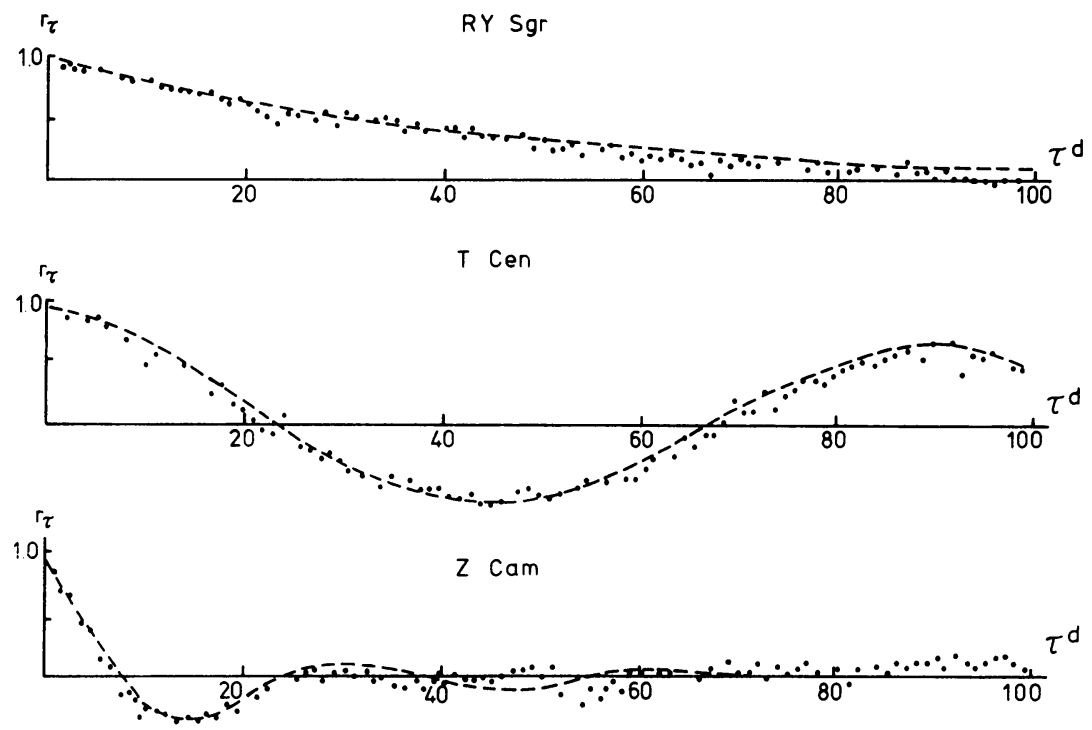

Fig. 1.

types in accordance with the results of Deeming (1970) (Figure 1). They are approximated by the formulae:

$$
\begin{aligned}
& r_{\tau}=A e^{-\tau / \tau_{0}}+B \cos \frac{2 \pi}{p} \tau+C \delta(\tau) \\
& r_{\tau}=A e^{-\left(\tau / \tau_{0}\right)^{2}}+B \cos \frac{2 \pi}{p} \tau+C \delta(\tau) \\
& r_{\tau}=A e^{-\tau / \tau_{0}} \cos \frac{2 \pi}{p_{1}} \tau+B \cos \frac{2 \pi}{p_{2}} \tau .
\end{aligned}
$$

These approximations are based on the assumption that the process of light variability is a sum of independent stochastic component processes. The terms in formulae 
(3) and their values by $\tau=0$ are the autocorrelative functions and the normalized dispersions of corresponding component processes.

The function $\delta(\tau)$ is introduced from the following considerations. According to formula (1), $r_{\tau=0}=1$. At the same time the sum of the values of the remaining terms in the first and second formulae can be much smaller for $\tau=0$. Therefore $C \delta(\tau)$ is an autocorrelative function of the component process and $\delta(0)=1$ while $\delta(\tau \neq 0)=0$. Such a component is a purely stochastic (random) process. It can be a white noise or a sequence of uncorrelated impulses. They origin at random moments and flare up and decline in a time smaller than a chosen time unit. Let us designate this process $Z(t)$.

A part of $C \delta(\tau)$ is given by observational errors $\left\{C^{\prime} \delta(\tau)\right\}$. The $C^{\prime}$ are estimated from the correlation:

$$
\frac{C^{\prime}}{1}=\frac{\sigma^{2}}{\overline{\left(m_{i}-\bar{m}\right)^{2}}}
$$

where $\sigma^{2}$ is a dispersion of errors. The difference $C-C^{\prime}$ is a normalized dispersion of the purely stochastic component of the process of variability. The other terms in formulae (3) are solutions of the equation

$$
a_{1} \frac{\mathrm{d}^{2} r_{\tau}}{\mathrm{d} \tau^{2}}+a_{2} \frac{\mathrm{d} r_{\tau}}{\mathrm{d} \tau}+r_{\tau}=0
$$

The common solution is

$$
r_{\tau}=N_{1} e^{K_{1} \tau}+N_{2} e^{K_{2} \tau},
$$

where $K_{1}$ and $K_{2}$ are the roots of the characteristic equation

$$
a_{1} K^{2}+a_{2} K+1=0
$$

and $N_{1}$ and $N_{2}$ are arbitrary constants.

$r_{\tau}=\cos (2 \pi / p) \tau$ when the roots are imaginary; $r_{\tau}=e^{-\tau / \tau_{0}} \cos (2 \pi / p) \tau$ when they are complex quantities; $r_{\tau}$ is a sum of two exponents when the roots are real and different; $r_{\tau}=\left(1+\tau / \tau_{0}\right) e^{-\tau / \tau_{0}}$ when they are real and equal. Such $r_{\tau}$ can be approximated by formula $e^{-\left(\tau / \tau_{0}\right)^{2}}$ also.

Equation (5) is satisfied by $r_{\tau}$ of processes $X(t)$ which satisfy the equation

$$
a_{1} \frac{\mathrm{d}^{2} X(t)}{\mathrm{d} t^{2}}+a_{2} \frac{\mathrm{d} X(t)}{\mathrm{d} t}+\{X(t)-X\}=Z(t) \text { (Deeming, 1970), }
$$

where $X(t)$ is determined by a correlation

$$
X_{t}=\alpha_{1} X_{t-1}+\alpha_{2} X_{t-2}+Z(t)
$$

$X(t)$ is modulated by a damping harmonic process perturbed by a white noise or by uncorrelated impulses. $r_{\tau}$ is equal to $e^{-\tau / \tau_{0}}$ for the process $Y(t)$ which satisfies the 
equation

$$
a \frac{\mathrm{d} Y(t)}{\mathrm{d} t}+\{Y(t)-\bar{y}\}=Z(t)
$$

Such processes are determined by a correlation

$$
Y_{t}=\alpha Y_{t-1}+Z(t)
$$

and are modulated by an exponentially-damped process perturbed by white noise or by a series of uncorrelated impulses.

$X(t)$ and $Y(t)$ are autoregressive processes of second and first order correspondingly.

Thus the autocorrelative analysis of magnitudes of an irregular or semi-regular variable star can be used for division of purely stochastic and unrandom perturbed component processes. It can also be used for determination of types of processes which modulate the process of variability.

Autocorrelative functions of the intensity of radiation were calculated for 15 variables also. They have the same types as the $r_{\tau}$ for series of magnitudes but with other values of parameters Lukatskaya (1969). Actually the same type of $r_{\tau}$ for intensity radiation and for series of magnitudes follows from Bochkov and Malachov (1974).

\section{References}

Bochkov, G. N. and Malachov, A. N.: 1974, Radiophys. 17, 693.

Campbell, L.: 1938, Annals Harvard Obs. 104.

Campbell, L.: 1940, Annals Harvard Obs. 107.

Campbell, L.: 1945, Annals Harvard Obs. 110.

Campbell, L.: 1948, Annals Harvard Obs. 116.

Deeming, T. I. : 1970, Astron. J. 75, 1027.

Kovalchuk, G. U., Lenderman, E. I., Lukatskaya, F. I., and Rosenbusch, A. E.: 1974, Col. Astronomia and Astrophysica, Main Astron. Obs. Ukr. SSR Acad. Sci. 25 (in press).

Lukatskaya, F. I.: 1969, A Statistical Study of Brightness of Irregular and Semi-Regular Variable Stars, Naukova dumka, Kiev.

Lukatskaya, F. I.: 1973, The Variable Stars, Moscow 19, 258. 